\title{
Pinning effects on the vortex critical velocity in type-II superconducting thin films
}

\author{
A. Leo ${ }^{\mathrm{a}, *}$, G. Grimaldi $^{\mathrm{a}}$, A. Nigro ${ }^{\mathrm{a}}$, S. Pace ${ }^{\mathrm{a}}$, N. Verellen ${ }^{\mathrm{b}}$, A.V. Silhanek ${ }^{\mathrm{b}}$, W. Gillijns ${ }^{\mathrm{b}}$, V.V. Moshchalkov ${ }^{\mathrm{b}}$, \\ V. Metlushko ${ }^{c}$, B. Ilic ${ }^{\mathrm{d}}$ \\ ${ }^{a}$ CNR-SPIN-Salerno and Dipartimento di Fisica “E R Caianiello", Universitá di Salerno, via Ponte Don Melillo, 84084 Fisciano (SA), Italy \\ ${ }^{\mathrm{b}}$ INPAC-Institute for Nanoscale Physics and Chemistry, KU Leuven, Celestijnenlaan 200D, Leuven B-3001, Belgium \\ ${ }^{\mathrm{c}}$ Department of Electrical and Computer Engineering, University of Illinois at Chicago, Chicago, IL 60607, USA \\ d Cornell Nanofabrication Facility, Cornell University, Ithaca, NY 14853, USA
}

\section{A R T I C L E I N F O}

\section{Article history:}

Available online 22 February 2010

\section{Keywords:}

Vortex dynamics

Vortex pinning

Magnetic pinning structures

\begin{abstract}
A B S T R A C T
We study the influence of artificial pinning centers on the vortex critical velocity in Al thin films deposited on top of a periodic array of Permalloy (FeNi) square rings. We demonstrate that the field dependence of the flux flow velocity strongly depends on the particular magnetic state of the rings. In particular, we find that, even when the rings are in a flux closure state, i.e. with little stray field, the vortex critical velocity shows a non-monotonic magnetic field dependence. This behaviour is in sharp contrast with the results obtained in a reference plain film, with no rings underneath. A comparison with the intrinsic strong pinning $\mathrm{Nb}$ films previously studied, suggests an interpretation in terms of a channel-like motion of vortices, here induced by the artificial pinning structure.
\end{abstract}

(c) 2010 Elsevier B.V. All rights reserved.

\section{Introduction}

The first model which describes the mechanism leading to the electronic flux-flow instability dates back to the 1980s. The starting point to study this phenomenon is the Larkin-Ovchinnikov theory [1], in which the presence of the instability is ascribed to the shrinking of the vortex core, as experimentally verified [2-4]. Indeed, the applied electric current sets the vortices in motion, but it can also give to the quasi-particles inside the vortex core enough energy to migrate out of the vortex core. Due to the finite quasiparticle energy relaxation rate $\tau_{\epsilon}^{-1}$, there could be such a velocity (the vortex critical velocity $v^{*}$ ) at which quasi-particles could not re-enter in the core and this leads to the reduction of the vortex diameter. These changes of the quasi-particle density inside and outside the vortex core are responsible for a dependence of the damping coefficient $\eta$ on vortex velocity, i.e. $\eta(v)=\frac{\eta(0)}{1+\sqrt{v / v^{*}}}$, with $\eta(0)$ the damping coefficient in the limit $v \rightarrow 0$ [5]. In turn, the dependence of $\eta$ on $v$ is responsible for the classical $\mathrm{N}$-shape of the voltage-biased current-voltage characteristics [6] and for the sudden jump from the flux-flow branch to the normal state by means of current driven measurements on type-II superconducting samples [2-4].

The assumptions made by the LO theory are quite restrictive. Indeed, this model is valid only near the superconducting critical temperature $T_{c}$ and in a window of applied magnetic fields such

\footnotetext{
* Corresponding author.

E-mail address: antoleo@sa.infn.it (A. Leo).
}

that the vortex can be considered as an isolated vortex (so the field has to be $\ll B_{c 2}$ ), but the quasi-particle distribution between vortices can be considered homogeneous (so the field has to be $\gg B_{c 1}$ ). Later on this model was upgraded by a few subsequent theoretical and several experimental studies.

From the theoretical side, the role of the heating effects was analyzed by Bezuglyj and Shklovskij [7]. They found that, even if the Joule heating is negligible, quasi-particles can experience an overheating due to the finite heat removal rate of the power dissipated in the sample which can trigger the instability. In the Bezuglyj-Shklovskij model, a magnetic field range is predicted in which the instability is triggered only by electronic effects. This regime takes place for $B>B_{T}$, where $B_{T}=\frac{0.347 e h \tau_{\epsilon}}{\sigma_{N} d}$ (here $\tau_{\epsilon}$ is the quasi-particle relaxation time, $\sigma_{N}$ and $d$ are the normal conductivity and the thickness of the sample). When $B<B_{T}$ quasi-particle overheating can be considered negligible.

Despite the prediction by the LO theory of a magnetic field independent vortex critical velocity $v_{0}^{*}$

$v_{0}^{*}=\sqrt{\frac{D[14 \zeta(3)]^{1 / 2}\left(1-T / T_{c}\right)^{1 / 2}}{\pi \tau_{\epsilon}}}$

where $D$ is the quasiparticle diffusion costant and $\zeta(n)$ the Riemann function, many experimental results show the existence of a crossover $B_{c r}$ between the field independent behavior and a field dependent one as the applied magnetic field $B$ is decreased $[5,8]$. The existence of this crossover can be explained considering that the quasiparticle distribution between the vortices is homogeneous if the distance $v^{*} \tau_{\epsilon}$ over which the quasi-particles themselves diffuse is 
larger than the inter-vortex distance $a_{0}$, which is well known to be field dependent $\left(a_{0}(B) \propto B^{-1 / 2}\right)$, thus [5]:

$v^{*}(B)=v_{0}^{*}\left(1+\frac{a_{0}(B)}{l_{\epsilon}}\right)$

where $l_{\epsilon}=\sqrt{\frac{1}{3} D \tau_{\epsilon}}$.

An important question that remains unsettled is: do the pinning properties of the material play a role in the electronic flux-flow instability? The early work by Ruck et al. [9] on Ta/Ge-based multilayers can be considered as a first attempt to answer this question. In this paper the authors observed LO type instabilities in the low and intermediate intrinsic pinning regimes and included in the $I-V$ equation a simple model of pinning forces which allowed them to capture many of the features of their experimental data. First of all, they explained the presence of a high non-linear fluxflow before the instability region due to the effect of the pinning. This is in contrast with the prediction of the LO theory, which predicts a linear free flux-flow before the bending of the curve which gives the typical N-shape to the $I(V)$ 's. Secondly, they also observed how increasing the pinning strength leads to a decreasing of the critical vortex velocities.

An interesting way to approach the problem of the interplay between pinning mechanism and instability phenomena would be the study of superconducting films with controlled artificial magnetic pinning structures (CAMPS). Indeed, it has been experimentally demonstrated how it is possible to tune the pinning strength by means of these CAMPS [10]. In this paper we present preliminary analysis on Aluminum (Al) thin films grown on top of a CAMPS made by a rectangular array of square micro-loops of Permalloy (Py, a Nickel-Iron magnetic alloy).

The particular choice of magnetic rings allows one to change from weak to strong pinning following a less lengthy and complicated procedure than in other CAMPS, as for example out-of-plane magnetized disks. In addition, the magnetic micro-loops give the chance to tune the pinning strength in a wider range respect to other CAMPS, as for example in-plane magnetized disks [11].

\section{Experimental results and discussion}

Here we show the results of investigations on samples which consist of a $50 \mathrm{~nm}$ thick Al film evaporated on top of a rectangular array of Py square micro-loops with a lateral size of $1 \mu \mathrm{m}$, a linewidth of $150 \mathrm{~nm}$, and thickness of $25 \mathrm{~nm}$ spaced by $100 \mathrm{~nm}$. The magnetic loops are electrically separated from the superconducting film by a $5 \mathrm{~nm}$ Si buffer layer added to reduce proximity effects. Both the micro-loops and the transport bridge were fabricated with electron-beam lithography and lift-off technique on a silicon substrate. The transport bridge has a width of $120 \mu \mathrm{m}$ and the distance $L$ between the voltage tips is $200 \mu \mathrm{m}$. The micro-loops are magnetized in such a way that they are in a flux-closure state (vortex state) [12]. A direct comparison between the critical current as function of the magnetic field, $I_{c}(H)$, in the vortex state and the $I_{c}(H)$ in the as-grown state has unambiguously demonstrated that the nature of the pinning interaction in the vortex state is magnetic $[13,14]$.

As a reference, a $50 \mathrm{~nm}$ thick transport bridge of $\mathrm{Al}$ with the same width and $L$ of the above cited bridge is taken. The Al films of the reference sample and the CAMPS sample are co-evaporated. The measurements are performed by a usual four-probe technique with the samples immersed in liquid Helium.

In Fig. 1 we show the voltage-current characteristics in a low voltage window of the plain film as a function of the applied magnetic field at a fixed temperature $t=T / T_{c}=0.89\left(T_{c}=1.36 \mathrm{~K}\right)$. In the inset of the same figure the $V(I)$ 's are shown in the whole range of measurement. The presence of a quite linear flux-flow branch

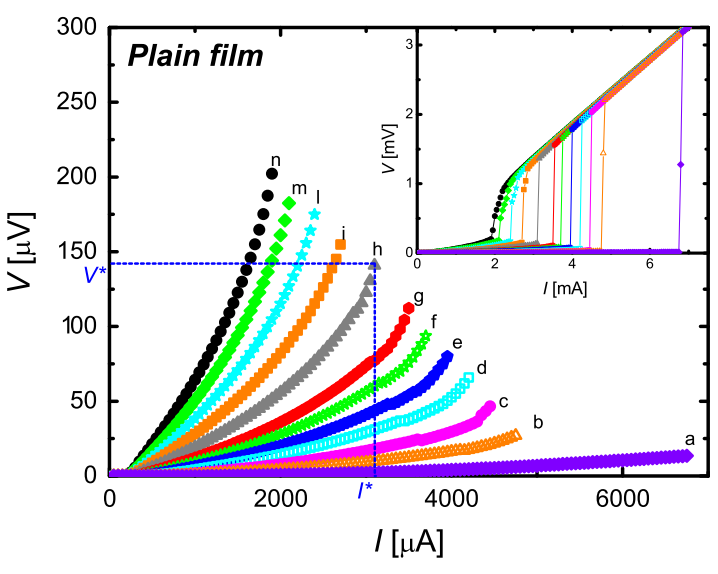

Fig. 1. $V(I)$ curves of the reference sample as a function of the applied magnetic field at $t=0.89$ : from $a=0.05 \mathrm{mT}$ to $g=0.30 \mathrm{mT}$ the magnetic field step is $0.05 \mathrm{mT}$, from $h=0.4 \mathrm{mT}$ to $n=0.8 \mathrm{mT}$ the magnetic field step is $0.1 \mathrm{mT}$. In the inset the curves are shown in the whole range of measurement.

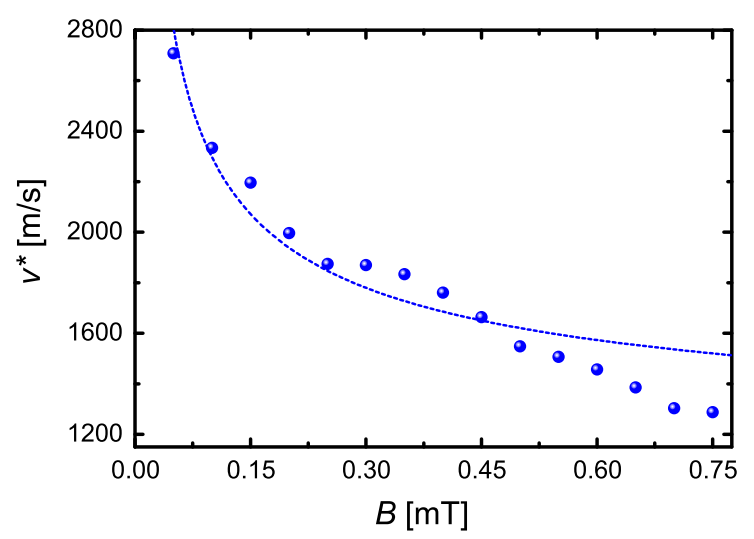

Fig. 2. The experimental $v^{*}(B)$ data at $t=0.89$ of the reference sample (blue spheres) together with the theoretical $v^{*}(B) \propto B^{-1 / 2}$ curve (dashed line). (For interpretation of the references to colour in this figure legend, the reader is referred to the web version of this article.)

followed by an upward bend and a sudden jump to the normal branch can easily be recognized. These features can be associated with the presence of LO instabilities. Indeed, we can exclude the possibility that the instabilities are triggered by heating effects. Indeed, from a thermal analysis, it results that the threshold value $B_{T}$ [15] is equal to $20 \mathrm{mT}$, much higher than the highest applied magnetic field. Moreover, the analysis of the dissipated power at the instability point $P^{*}=I^{*} V^{*}$ (where $I^{*}$ and $V^{*}$ are, respectively, the current and the voltage values at the jump point to the normal state) shows a clear dependence on the applied magnetic field. As reported by Xiao et al. [16], this is experimental evidence that the instability is not due to Joule self-heating effects.

Another confirmation of the electronic instability nature comes from the extrapolated critical vortex velocity dependence on the applied magnetic field $v^{*}(B)$. Indeed, in Fig. 2 we show the experimental $v^{*}(B)$ curve as obtained from the data of Fig. 1. The critical vortex velocities are deduced with the formula $v^{*}=V^{*} / B L$. The figure shows how the $v^{*}$ data approximately follow the expected $B^{-1 / 2}$ behavior (dashed line).

Fig. 3 shows the $V(I)$ curves of the sample with the micro-loops in the vortex state, i.e. with a stronger pinning, as a function of the applied magnetic field still at $t=0.89$. Again, the main panel is related to the low voltage window, while in the inset the data are related to the whole range of measurement. These curves are highly 


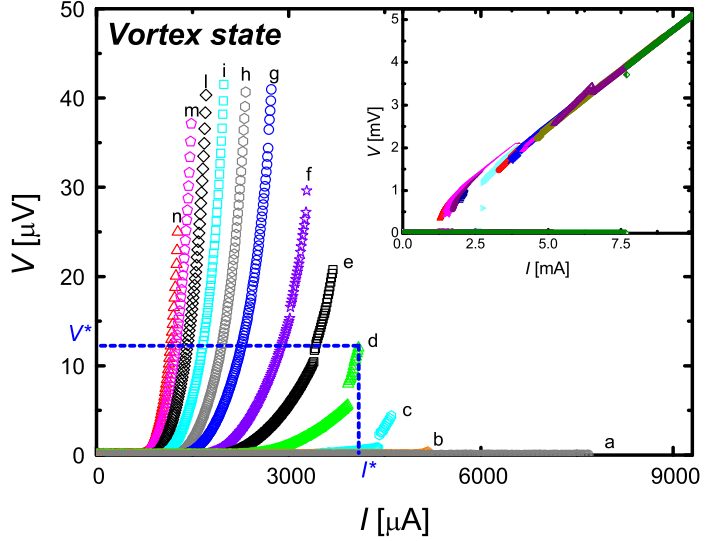

Fig. 3. $V(I)$ curves of the sample with CAMPS in the vortex state as a function of the applied magnetic field at $t=0.89$ : from $a=0 \mathrm{mT}$ to $f=0.5 \mathrm{mT}$ the magnetic field step is $0.1 \mathrm{mT}$, from $g=0.7 \mathrm{mT}$ to $n=1.7 \mathrm{mT}$ the magnetic field step is $0.2 \mathrm{mT}$. In the inset the curves are shown in the whole range of measurement.

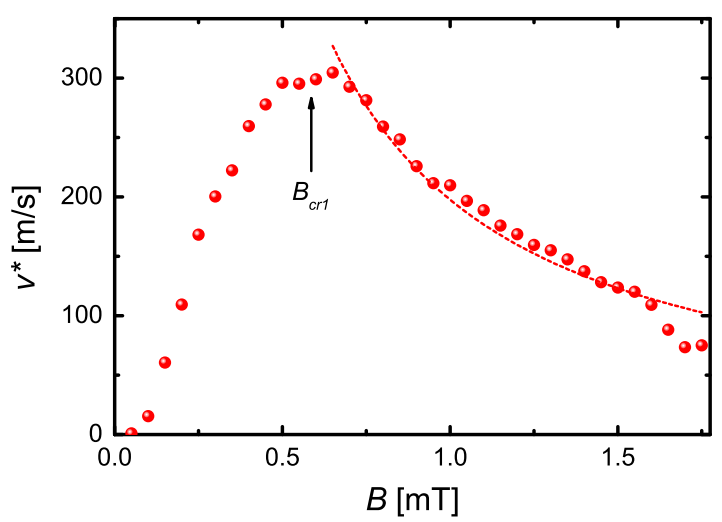

Fig. 4. The experimental $v^{*}(B)$ data at $t=0.89$ of the sample with CAMPS in the vortex state (red spheres) together with the theoretical $v^{*}(B) \propto B^{-1 / 2}$ curve (dashed line). (For interpretation of the references to colour in this figure legend, the reader is referred to the web version of this article.)

non-linear with respect to the reference sample characteristics (see Fig. 1). This is in agreement with the cited observations by Ruck et al. [9]. Moreover, in Fig. 4 the experimental $v^{*}(B)$ curve of the sample with the CAMPS shows critical vortex velocity values lower than the $v^{*}$ 's values of the plain film, again in agreement with the previous results [9].

In Fig. 4 the presence of a new low field crossover $B_{c r 1}$ from a monotonic increasing of $v^{*}$ from $B=0$ to the usual expected power law behavior $B^{-1 / 2}$ is shown. This original experimental result has been recently connected to a channel-like vortex motion due to a fan-like penetration of the vortices in the moderately strong pinning $\mathrm{Nb}$ films $[17,18]$. Here the channel-like vortex motion is instead sustained by the artificial pinning structures.

\section{Conclusions}

In conclusion, we present preliminary results on Al thin films grown on top of magnetic micro-loops with tunable pinning capabilities. We can confirm that the pinning strength strongly influences the vortex dynamics, particularly the critical vortex velocity behavior. Indeed, the value of $v^{*}$ decreases as the pinning strength increases and, more interesting, it results in an original critical vortex velocity dependence on the applied magnetic field induced by the channel-like vortex motion through a controlled artificial magnetic pinning structure.

\section{Acknowledgments}

This work was supported by the Research Project L.R. No. 5, Regione Campania, Methusalem Funding of the Flemish government, FWO-Vlaanderen, the Belgian Inter-University Attraction Poles IAP, the ESF-NES Programs, and the US NSF, grant ECCS0823813 (VM). A.L. acknowledges the funding support under the contract CO 03/2009. A.V.S. and W.G. are grateful for the support from the FWO-Vlaanderen.

\section{References}

[1] A.I. Larkin, Yu.N. Ovchinnikov, Zh. Eksp. Teor. Fiz. 68 (1975) 1915.

[2] W. Klein, R.P. Huebener, S. Gauss, J. Parisi, J. Low Temp. Phys. 61 (1995) 413.

[3] G. Grimaldi, A. Leo, A. Nigro, S. Pace, R.P. Huebener, Phys. Rev. B 80 (2009) 144521.

[4] D. Babic, J. Bentner, C. Sürgers, C. Strunk, Phys. Rev. B 69 (2004) 092510.

[5] S.G. Doettinger, R.P. Huebener, A. Kühle, Physica C 251 (1995) 285.

[6] A. Wehner, O.M. Stoll, R.P. Huebener, M. Naito, Phys. Rev. B 63 (2000) 144511.

[7] A.I. Bezuglyj, V.A. Shklovskij, Physica C 202 (1992) 234

[8] G. Grimaldi, A. Leo, C. Cirillo, C. Attanasio, A. Nigro, S. Pace, J. Phys.: Condens. Matter 21 (2009) 254207.

[9] B.J. Ruck, H.J. Trodahl, J.C. Abele, M.J. Geselbracht, Phys. Rev. B 62 (2000) 12468

[10] A.V. Silhanek, W. Gillijns, V.V. Moshchalkov, V. Metlushko, B. Ilic, Appl. Phys. Lett. 89 (2006) 182505.

[11] [11]A.V. Silhanek, N. Verellen, V. Metlushko, W. Gillijns, F. Gozzini, B. Ilic, V.V. Moshchalkov, Physica C 468 (2008) 563.

[12] P. Vavassori, M. Grimsditch, V. Novosad, V. Metlushko, B. Ilic, Phys. Rev. B 67 (2003) 134429.

[13] N. Verellen, A.V. Silhanek, W. Gillijns, V.V. Moshchalkov, V. Metlushko, F. Gozzini, B. Ilic, Appl. Phys. Lett. 93 (2008) 022507.

[14] N. Verellen, A.V. Silhanek, W. Gillijns, V.V. Moshchalkov, V. Metlushko, F. Gozzini, B. Ilic, Appl. Phys. Lett. 94 (2009) 219903.

[15] G. Grimaldi, A. Leo, A. Nigro, S. Pace, A. Angrisani Armenio, C. Attanasio, J. Phys.: Conf. Ser. 97 (2008) 012111.

[16] Z.L. Xiao, P. Voss-de Haan, G. Jakob, Th. Kluge, P. Haibach, H. Adrian, E.Y. Andrei, Phys. Rev. B 59 (1999) 1481

[17] G. Grimaldi, A. Leo, A. Nigro, S. Pace, C. Cirillo, C. Attanasio, Physica C 468 (2008) 765 .

[18] G. Grimaldi, A. Leo, D. Zola, A. Nigro, S. Pace, F. Laviano, E. Mezzetti, Phys. Rev. $\mathrm{B}$, submitted for publication. 